\title{
Prevalencia de trastornos músculo-esqueléticos en el personal de esterilización en tres hospitales públicos
}

\author{
Prevalence of musculoskeletal disorders in the sterilization staff in three public \\ hospitals
}

\author{
Rosa María Rosario Amézquita ${ }^{1}$, Teresa Isabel Amézquita Rosario ${ }^{2}$ \\ 1. Hospital San Pedro. Logroño. España. \\ 2. Instituto de Salud Pública y Laboral. Navarra. España. \\ Recibido: 16-10-13 \\ Aceptado: 17-02-14

\section{Correspondencia} \\ Rosa M. ${ }^{a}$ Rosario Amézquita \\ C/12 Ligero de Artillería 35, 2-A \\ 26004 Logroño. España \\ rm.amezquita@hotmail.com \\ Teléfono 638131078
}

Resumen

Introducción: Los trastornos músculo-esqueléticos, según informe de la agencia Europea para la Seguridad y Salud en el Trabajo, constituyen el problema de salud laboral más común en Europa.

Objetivo: Identificar la prevalencia de trastornos músculo-esqueléticos en trabajadores de esterilización de tres hospitales de España.

Metodología: Se realizó un estudio descriptivo transversal en las empleadas del Servicio de esterilización de tres hospitales de Rioja y Navarra mediante la utilización del cuestionario nórdico de I. Kuorinka, en el que se identifican datos socio-demográficos y se registran síntomas músculo-esqueléticos. Para identificar la exposición a factores de riesgos ergonómicos y psicosociales se utilizaron las preguntas propuestas para la revisión de la Encuesta de Salud y Condiciones de Trabajo de España. Los datos se analizaron utilizando el programa estadístico SPSS versión 17.

Resultados: Entre 38,3-57,3\% de las trabajadoras dicen estar expuestas a riesgos ergonómicos a lo largo de toda la jornada de trabajo. Los riesgos psicosociales alcanzan frecuencias mayores. El mayor porcentaje de molestias músculo-esqueléticas en los 7 días previos a la cumplimentación del cuestionario se presentaron en cuello $(71,7 \%)$, seguido de las molestias de espalda $(59,1)$.

Discusión: Respecto a población general, en nuestro estudio son mayores las frecuencias de trabajadoras expuestas a ritmos elevados de trabajo y realización de tareas muy repetitivas de corta duración. También se observa la importancia de algunas de las variables psicosociales a la hora de explicar la aparición de los daños ergonómicos.

Conclusiones: Sería oportuna la realización en este colectivo de la evaluación de riesgos de movimientos repetidos en extremidad superior con métodos validados. Las exposiciones ergonómicas y psicosociales identificadas aconsejan actuaciones preventivas.

Med Segur Trab (Internet) 2014; 60 (234) 24-43

Palabras clave: Trastornos músculo-esqueléticos, Riesgo ergonómico, Servicio de esterilización. 


\section{Abstract}

Introduction: Musculo-skeletal disorders, as reported by the European Agency for Safety and Health at Work, are the occupational health problem more common in Europe. They are injuries of muscles, tendons, nerves and joints.

Objective: To identify the prevalence of musculo-skeletal disorders in sterilization workers in three hospitals in Spain.

Methods: We performed a cross-sectional study involving employees of the sterelization unit in three hospitals of Rioja and Navarra by using the Nordic questionnaire I. Kuorinka, which socio-demographic data can be identified and a second that allows to record musculo-skeletal symptoms. To identify exposure to ergonomic risk factors and psychosocial questions were used to review proposals from the Survey of Health and Working Conditions of Spain. The data was analyzed using SPSS version 17.

Results: Between 38.3 to $57.3 \%$ of workers say they are exposed to ergonomic hazards throughout the working day. Psychosocial risks reach higher frequencies. The highest percentage of musculoskeletal discomfort in the 7 days prior to the questionnaire were presented in neck ( $71.7 \%)$, followed by back (59.1).

Discussion: Regarding general population in our study workers exposed to high rates of work and highly repetitive tasks of short duration show higher frequencies. It also notes the importance of some of tasks of the psychosocial variables in explaining the emergence of ergonomic injury.

Conclusions: It would be opportune to perform in this group of risk assessment of upper extremity repetitive movements with validate methods. Ergonomic and psychosocial exposures advise preventive actions identified.

Med Segur Trab (Internet) 2014; 60 (234) 24-43

Keywords: musculo-skeletal disorders, ergonomic risk, sterilization services 


\section{INTRODUCCIÓN}

En nuestra revisión bibliográfica no hemos hallado publicado estudio alguno de LME referido al personal de los servicios de esterilización hospitalario a pesar de que la descripción de sus tareas permite identificar la existencia de peligro de LME.

Los trastornos músculo-esqueléticos son lesiones de músculos, tendones, nervios y articulaciones que se localizan más frecuentemente en el cuello, espalda, hombros, codos, muñecas y manos. El síntoma predominante es el dolor, asociado a inflamación, pérdida de fuerza y dificultad o imposibilidad para realizar algunos movimientos. Este grupo de enfermedades se da con gran frecuencia en trabajos que requieren una actividad física importante, movilización de pesos, movimientos repetidos o aplicación de fuerzas y también aparece en otros trabajos como consecuencia de malas posturas sostenidas durante largos periodos de tiempo ${ }^{1}$.

Los trastornos músculo-esqueléticos (TME), según informe de la Agencia Europea para la Seguridad y Salud en el Trabajo, son el problema de salud laboral más común en la Europa de los veintisiete (EU-27), donde el 25\% de los trabajadores Europeos se quejan de dolores de espalda, y el $23 \%$ de dolores musculares, en el último año previo a la realización de la encuesta. ${ }^{2}$

Citamos a continuación algunas de las numerosas patologías incluidas bajo esta denominación ${ }^{3}-5$.

Síndrome del túnel carpiano, Tendinitis, Tenosinovitis, Síndrome del canal cubital, Epicondilitis, Epitrocleítis, Síndrome del túnel radial, Síndrome del pronador redondo, Roturas de fibras Esguinces, Bursitis, Artrosis, Artritis, Hernias discales, Fracturas, Síndrome cervical por tensión.

Ya a comienzo del siglo XVIII, se conocía la relación de los TME con factores etiológico ocupacionales, pero no fue hasta la década de 1970 que estos factores ocupacionales se analizaron mediante métodos epidemiológicos, apareciendo ya regularmente en la literatura científica internacional ${ }^{4}$.

Según los datos de la II Encuesta Navarra de salud y condiciones de trabajo del año 2006, última realizada, el $48 \%$ de los trabajadores presenta alguna molestia músculo-esquelética derivada de posturas y esfuerzos en el trabajo, siendo la de zona lumbar con un 39\% la más frecuente, seguida de la nuca/cuello con un 30\% y alto de espalda con un $26 \%$. Las molestias en hombros, brazos y manos/muñecas presentan frecuencias del 16\%, el 13\% y el $12 \%$ respectivamentes.

En España, las cifras son similares. Según la VI Encuesta Nacional de Condiciones de Trabajo (VI-ENCT) realizada en 2007 , el 74,2\% de los trabajadores encuestados señala sentir alguna molestia que achaca a posturas y esfuerzos asociados al trabajo que realiza. Entre las molestias más frecuentes, figuran las localizadas en la zona baja de espalda (40,1\%), la nuca/cuello (27\%) y la zona alta de espalda $(26,6 \%)^{6}$. Datos similares pueden verse en Alemania, Finlandia, EE. UU, Suecia, Inglaterra y Colombia ${ }^{7,8}$.

Centrándonos ya entre los trabajadores de la actividad de salud, un estudio en profesionales sanitarios realizado en las Isla Baleares en España revela que el 59,3\% de los trabajadores presentó dolor de espalda, el 26,7\% dolor cervical, el 22,1\% dolor lumbar, dolor en las piernas y brazos el $7 \%$ y un $24,4 \%$ padecía dolor en rodillas, hombro y muñecas, en el momento de la entrevista9.

Estudios realizados en enfermería hospitalaria señalan que la prevalencia de los trastornos músculo-esqueléticos, en concreto el dolor de espalda se sitúa en torno al $80-85 \%{ }^{10}$.

Otros estudios realizados en Europa y América del Norte muestran que el personal de enfermería es un colectivo particularmente afectado por el dolor de espalda si se compara con otros grupos profesionales o sectores de actividad económica. Los auxiliares 
de clínica son el grupo con más alta prevalencia, con un riesgo de 2,2 veces superior de padecer lumbalgia que la población general ${ }^{11}$.

Varios de los TME están incluidos en el cuadro de Enfermedades Profesionales como los provocados por posturas forzadas y movimientos repetidos: tendinitis del manguito de los rotadores, epicondilitis y epitrocleítis, tendinitis del abductor largo y extensor corto del pulgar, tenosinovitis estenosante digital, tenosinovitis del extensor largo del primer dedo, síndrome del túnel carpiano y el síndrome del canal de Guyón 5.

Destaca el subregistro de estas patologías en todo el Estado español si consideramos la incidencia de casos en Navarra. En el 2010, la declaración en Navarra fue de 677,5 por 100,000 trabajadores $^{12}$ frente a la declarada en España que fue de 116,21 por 100,000 trabajadores $^{13}$.

\section{HIPÓTESIS Y OBJETIVOS}

La prevalencia de los trastornos músculo-esqueléticos (TME) puede ser mayor en los trabajadores de esterilización que en la población general.

\section{Objetivo General}

Identificar la prevalencia de trastornos músculo-esqueléticos en trabajadores de esterilización de tres hospitales de España, año 2011-2012.

\section{Objetivo específicos}

Caracterizar los trabajadores con trastornos músculo-esqueléticos según variable socio-demográficos.

Explorar la asociación entre el daño percibido y variables identificadas de exposición ergonómica y psicosocial.

\section{Población, material y método}

Este trabajo es un estudio descriptivo transversal sobre daño músculo esquelético percibido e identificación de exposición a riesgos ergonómicos y psicosociales realizado entre las trabajadoras de las unidades de esterilización de dos hospitales públicos.

Nuestra población está formada por el total de trabajadores del servicio de esterilización de los hospitales públicos Complejo hospitalario de Navarra (Hospital A y Hospital B) y Hospital San Pedro (La Rioja).

\section{Descripción de las tareas}

Esta descripción se realiza tras entrevista con las personas responsables de los servicios incluidos en el estudio. Las tareas realizadas en el servicio de esterilización consisten en la recepción de material que envían de los quirófanos, lavado manual de instrumental, carga y descarga de lavadoras de carros, carga y descarga de lavadoras automáticas (incluye baños de ultrasonidos), embolsado y termosellado, empaquetado de cajas contenedoras y equipos de material textil, carga de los autoclaves de vapor, gas plasma y oxido de etileno, descarga de los autoclaves de gas, plasma y vapor, almacenamiento de material en estanterías, carga de contenedores en los carros para el día siguiente y suministro de material en caso de urgencias.

Todas las tareas se realizan en bipedestación, algunas excepcionalmente se pueden realizar en silla de asiento alto.

Se tiene establecido un régimen de rotación de tareas el cual consiste en la ejecución de una tarea durante toda una semana volviendo a repetirla cuatro o cinco semanas después, es decir, que una tarea se realiza una semana al mes, aproximadamente. 
No hay distintas categorías profesionales u ocupaciones, ni están asociadas las ocupaciones al nivel de estudios o a la antigüedad en la empresa o en el servicio.

En el complejo hospitalario de Navarra "B", anterior Hospital Virgen del camino, no se lavan los instrumentos, llegan limpios al servicio de esterilización.

De esta descripción de tareas podemos identificar riesgos ergonómicos asociados a movimientos repetidos, posturas mantenidas o forzadas, manipulación manual de cargas, aplicación de fuerzas etc. También la exposición a factores psicosociales laborales puede jugar un importante papel en la explicación de la aparición de daños músculoesqueléticos.

\section{Criterios de inclusión y exclusión}

\section{Criterios de inclusión}

El estudio se lleva a cabo en el personal que trabaja en la unidad de esterilización en el período de estudio.

\section{Criterios de exclusión}

Trabajadores que presenten lesiones musculares antes de empezar a trabajar en la central de esterilización y los que tengan lesiones músculo-esqueléticas de origen congénito. No se ha considerado como criterio de exclusión la escasa antigüedad en la unidad dado que algunas de las patologías derivadas de exposiciones a factores ergonómicos de riego pueden desarrollarse en un corto intervalo de tiempo.

\section{Variables estudiadas}

- De identificación:

- Sexo.

- Edad: Hemos categorizado la edad en menor o iguala 45 años y mayor de 45 años.

- Centro de trabajo: Al inicio de la investigación en Navarra existían dos hospitales incluidos en el estudio. Posteriormente fueron unificados.

- Categoría ocupacional. Todas las personas del estudio tienen la misma categoría luego esta variable no aparece en la descripción de resultados.

- Variables psicosociales:

Todas ellas se refieren a la percepción que tiene la trabajadora sobre las mismas.

- Nivel de atención requerido por la tarea.

- Autonomía de la trabajadora en cuanto a la realización del orden de las tareas, en cuanto a tomar decisiones en la organización de su trabajo, etc.

- Ritmo de trabajo.

- Control del trabajo: Incluye conceptos de participación a la hora de la organización del trabajo.

- Necesidad de esconder emociones.

- Apoyo social tanto de compañeras como de superiores en la vertiente de hacer frente al trabajo y también respecto a la vertiente de considerarse respetada personalmente.

- Variables ergonómicas:

- Manipulación manual de cargas.

- Realización de movimientos repetidos.

- Adopción de posturas forzadas.

- Aplicación de fuerza. 
- Variable de efecto

Molestia ósteo articular o muscular en los últimos 7 días.

\section{Trabajo de campo}

Las encuestas se cumplimentaron en los meses de junio y julio de 2011.

En el desarrollo de la investigación en una primera fase tuvimos una reunión con la supervisora y algunas de las trabajadoras de la unidad de esterilización del Complejo Hospitalario de Navarra (A y B) y del Hospital San Pedro en Logroño. Comunicamos los objetivos y el interés de la realización de un estudio de prevalencia de los trastornos músculo-esqueléticos en ese colectivo y la importancia de conocer los riesgos más prevalentes, para así poder proponer recomendaciones y actuaciones preventivas.

Les informamos que las encuestas serían anónimas. Les solicitamos autorización y nos dijeron que era preferible dejar los cuestionarios en la Unidad y que cada supervisora se encargaría de entregar los cuestionarios a cada trabajadora ya que algunas estaban de vacaciones. Le entregamos los cuestionarios a la supervisora. A las dos semanas fueron recogidas las encuestas ya cumplimentadas.

\section{Recogida y análisis de datos}

Para identificar la exposición hemos utilizado las variables del cuestionario sobre condiciones de trabajo y salud revisado por F. G. Benavides et al., referidas a factores de riesgo ergonómicos y psicosociales ${ }^{7}$.

Para detectar las molestias músculo-esqueléticas hemos usado el cuestionario nórdico estandarizado de I. Kuorinka y cols, validado en España en el año 2000 el cual consta de dos partes, una primera que se interesa por datos socio demográficos tales como antecedentes personales y actividad laboral y una segunda que permite registrar síntomas músculo-esqueléticos en 9 segmentos corporales (cuello, hombros, codos, muñecas/manos, espalda superior, espalda inferior, cadera/muslo, rodillas y tobillos), evaluados en 3 dimensiones como son restricción motora, frecuencia del dolor y localización del mismo durante dos períodos, últimos 3 meses y los 7 días precedentes ${ }^{14,15}$.

Los datos se analizaron utilizando el programa estadístico SPSS versión 17 comparando la prevalencia de daño según categorías de las variables sociodemográficas y de exposición incluidas en el cuestionario.

\section{Resultados}

Fueron encuestados 56 trabajadores en los tres hospitales públicos: Complejo hospitalario de Navarra (A y B), anteriormente hospital de Navarra y hospital Virgen del camino y Hospital San Pedro (La Rioja). Participó el 100\% de la planilla, siendo 55 personas de sexo femenino y una de sexo masculino. Este último es excluido de los análisis posteriores, ya que son conocidas las diferencias antropométricas y funcionales entre $\operatorname{sexos}^{16}$.

Vemos en la tabla I que el número de trabajadoras de este servicio es similar en ambas regiones estudiadas. Destaca la elevada temporalidad en los Servicios de esterilización alcanzando esta más del $45 \%$ de la población a estudio. La mayor parte de la plantilla trabaja a turno rotatorio alternando mañana y tarde. 
Tabla I. Descripción sociodemográfica de la población

\begin{tabular}{lcclcc}
\hline & $\mathbf{N}$ & $\%$ & & $\mathbf{N}$ & $\%$ \\
\hline Edad (años) & & & Contrato & & \\
$<45$ & 22 & 40,0 & Fijo & 30 & 54,5 \\
$>45$ & 33 & 60,0 & Temporal & 25 & 45,5 \\
\hline Nivel educativo (\%) & & & Horario & 8 & 14,5 \\
\hline ESO & 9 & 16,7 & Jornada Partida & 8 & 14,5 \\
Bachiller & 33 & 61,1 & Fijo Mañana & 3 & 5,5 \\
Diplomatura/superior & 12 & 22,2 & Fijo Tarde & 34 & 61,8 \\
& & & Rotatorio sin noches & & \\
\hline Centro de trabajo & & & & & \\
\hline Virgen del Camino & 10 & 18,2 & & & \\
Hospital de Navarra & 18 & 32,2 & & & \\
Hospital San Pedro & 27 & 49,1 & & & \\
\hline
\end{tabular}

La edad media de la población estudiada fue de 47 años. Al comparar las medias de edades entre ambos hospitales vemos que las trabajadoras del Complejo hospitalario de Navarra (50.07 años SD: 8.92) son significativamente mayores que las de Logroño (43.81 años SD: 6.66) [test Student: $\mathrm{t}<0.005$ ].

El test Student es una distribución de probabilidad que surge del problema de estimar la media de una población normalmente distribuida cuando el tamaño de la muestra es pequeño.

Permite comprobar si es posible aceptar que la media de la población es un valor determinado. Se toma una muestra y el Test permite evaluar si es razonable mantener la Hipótesis nula de que la media es tal valor ${ }^{29}$.

Respecto a la antigüedad en las tareas según nos informan las medianas correspondientes, en torno a la mitad de la plantilla tiene más de 5 años de antigüedad en el puesto de trabajo siendo este valor de 14 años en cuanto a la antigüedad en la empresa.

En la tabla II al observar las variables de exposición ergonómicas vemos que entre el 38,3 y el 57,4\% de las trabajadoras dicen estar expuestas a riesgos ergonómicos durante toda la jornada de trabajo. Entre los riesgos destaca la frecuencia de exposición a manipulación manual de cargas y a movimientos repetidos. El 91,5\% de la plantilla al primer factor de riesgos citado y el 85,1 al segundo con unas frecuencias de siempre o muchas veces.

Tabla II. Frecuencia (\%) de exposición a factores de riesgo ergonómicos

\begin{tabular}{lccc}
\hline \multicolumn{1}{c}{ Exposición Ergonómica } & Siempre & Muchas veces & Alguna vez o nunca \\
\hline Manipulación Manual Cargas & 53,2 & 38,3 & 8,5 \\
Movimientos Repetidos & 57,4 & 27,7 & 14,9 \\
Posturas Forzadas & 38,3 & 23,4 & 38,3 \\
Aplicación Fuerzas & 40,4 & 34,0 & 25,5 \\
\hline
\end{tabular}

Observamos en la tabla III que las trabajadoras perciben estar expuestas particularmente a un exceso de carga de trabajo asociada a la atención requerida por la tarea, las presiones de tiempo, identificadas por el trabajo muy rápido y el tiempo insuficiente, y a la ausencia de autonomía a la hora de organizar su propio trabajo o al no poder poner en práctica sus propias ideas. Sienten también contar con escaso apoyo social, tanto de compañeras como de supervisoras, e identifican estar expuestas a riesgos ergonómicos asociado al diseño del puesto de trabajo que les limitan el trabajar con comodidad, la libertad de movimientos, el no tener que forzar la vista y el adoptar posturas adecuadas. 
Tabla III. Frecuencia de trabajadoras expuestas (\%) a diversos tipos de factores de riesgo psicosocial

\begin{tabular}{|c|c|c|}
\hline Riesgo & $\begin{array}{c}\text { Muchas veces/ } \\
\text { Siempre }\end{array}$ & $\begin{array}{l}\text { Alguna vez/ } \\
\text { nunca }\end{array}$ \\
\hline Requerimiento atención alto o muy alto & 80,9 & \\
\hline Atención varias tareas al mismo tiempo & 59,6 & \\
\hline Realización tareas complejas o difíciles & 38,3 & \\
\hline Necesidad esconder emociones & 27,7 & \\
\hline Trabajo excesivo & 31,9 & \\
\hline Trabajo muy rápido & 55,3 & \\
\hline Plazos muy cortos & 36,2 & \\
\hline Tiempo insuficiente & 72,3 & \\
\hline Los horarios los fija la empresa & 57,4 & \\
\hline \multicolumn{3}{|l|}{ Frecuencia en la que el espacio le permite } \\
\hline - Trabajar cómodo & & 44,7 \\
\hline - Libertad de movimientos & & 53,2 \\
\hline - Autonomía postural & & 38,3 \\
\hline \multicolumn{3}{|l|}{ Frecuencia en la que la iluminación le permite } \\
\hline - Trabajar con postura adecuada & & 40,4 \\
\hline - No forzar la vista & & 44,7 \\
\hline Recibe información clara y suficiente & & 12,8 \\
\hline Recibe adiestramiento & & 48,9 \\
\hline Libertad de vacaciones/días libres & & 25,5 \\
\hline Oportunidad de hacer lo que sabe hacer & & 53,2 \\
\hline Pone en práctica sus ideas & & 61,7 \\
\hline \multicolumn{3}{|l|}{ Disponibilidad de autonomía } \\
\hline - En el orden de las tareas & & 58,7 \\
\hline - En el método de trabajo & & 73,9 \\
\hline - En el ritmo de trabajo & & 70,2 \\
\hline - En la pausas & & 68,1 \\
\hline Recibe ayuda de compañeros & & 29,0 \\
\hline Recibe ayuda de supervisores & & 29,0 \\
\hline Relaciones personales positivas & & 34,0 \\
\hline
\end{tabular}

En la tabla IV Considerando el conjunto de las molestias hallamos una frecuencia del $78.2 \%$ de trabajadores que presentaban alguna molestia músculo-esqueléticas (MME) en los últimos 7 días. No parece haber diferencias entre las frecuencias de daños entre los tres meses previos y los 7 días anteriores a la cumplimentación del cuestionario. Con objeto de minimizar el posible sesgo de memoria a partir de ahora cuando hablemos de daño nos referimos al identificado para la última semana. 


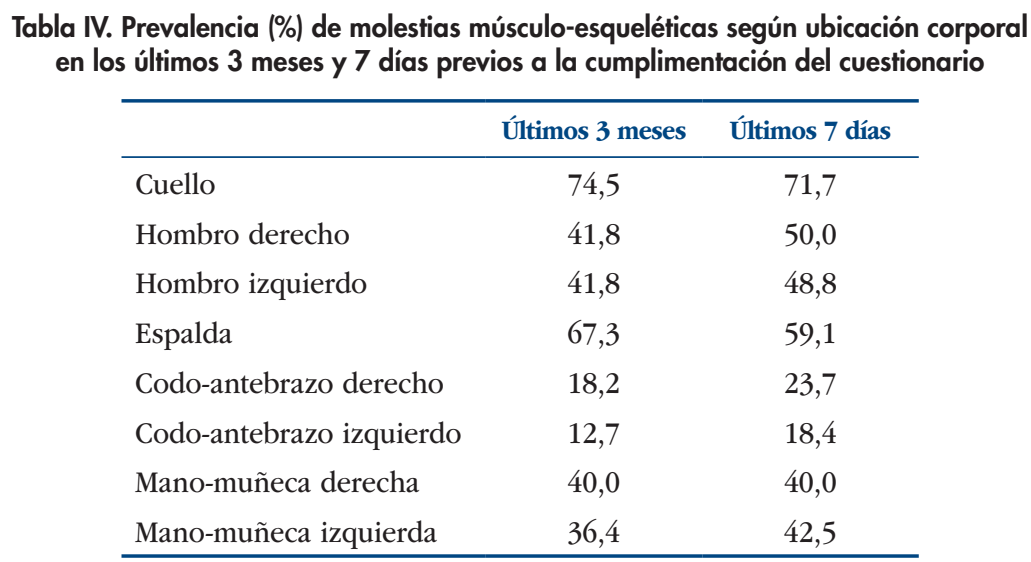

En nuestro estudio el mayor porcentaje de MME se presentaron en el cuello, con un $70 \%$ de afectadas, seguido de la espalda con el 60\% de dañadas, el segmento muñecamano con un $40 \%$ de empleadas que perciben molestias y el menor porcentaje se da en las molestias en codo-antebrazo, con entre el 18 y el $24 \%$ de afectadas.

Vemos en la tabla V que el dolor de cuello y espalda es más frecuente en menores de 45 años, que el horario a turnos parece aumentar el riesgo de molestias en cuello u hombro derecho y que en el Complejo hospitalario de Navarra son más frecuentes las molestias de hombro y antebrazo izquierdo. En el resto de variables sociodemográficas no hay diferencias estadísticamente significativas en la distribución de las frecuencias según sus categorías.

Tabla V. Distribución del daño según variables de identificación sociodemográficas

\begin{tabular}{|c|c|c|c|c|c|c|c|c|c|c|}
\hline & \multicolumn{2}{|c|}{ Cuello } & \multicolumn{2}{|c|}{ Espalda } & \multicolumn{2}{|c|}{ Hombro derecho } & \multicolumn{2}{|c|}{ Hombro izquierdo } & \multicolumn{2}{|c|}{$\begin{array}{c}\text { Codo Antebrazo } \\
\text { izquierdo }\end{array}$} \\
\hline & $\%$ & $\mathbf{p}$ & $\%$ & $\mathbf{p}$ & $\%$ & $\mathbf{p}$ & $\%$ & $\mathbf{p}$ & $\%$ & $\mathbf{p}$ \\
\hline \multicolumn{11}{|l|}{ Edad } \\
\hline$<45$ & 90,9 & 0,02 & 86,4 & 0,01 & & & & & & \\
\hline$>45$ & 63,6 & & 54,5 & & & & & & & \\
\hline \multicolumn{11}{|l|}{ Horario } \\
\hline Fijo & 50,5 & 0,02 & & & 29,4 & 0,02 & & & & \\
\hline Turnos & 85,7 & & & & 64,0 & & & & & \\
\hline \multicolumn{11}{|l|}{ Hospital } \\
\hline Navarra & & & & & & & 86,7 & 0,00 & 41,7 & 0,22 \\
\hline S. Pedro & & & & & & & 26,9 & & 7,7 & \\
\hline
\end{tabular}

\section{Discusión}

Como limitación del estudio hay que señalar que, como en todo estudio transversal, no es posible establecer el sentido de las asociaciones observadas entre las variables de exposición y de daño, entre la posible causa y su efecto.

Pudiera haber existido sesgo en los resultados debido a las no respuestas de trabajadoras en situación de Incapacidad Temporal asociada a daño ergonómico en expuestos. No obstante en el momento de la encuesta no había trabajadora alguna en situación de incapacidad temporal.

Desconocemos si entre las razones que explican la diferencia de antigüedad en la empresa respecto a la antigüedad en el servicio de esterilización se encuentran motivos ergonómicos. 
Tampoco ha sido considerado el hábito tabáquico de las empleadas en nuestro estudio pese a que hay trabajos que muestran un aumento de riesgo de dolor músculo esquelético en todas las localizaciones tanto en fumadores como en exfumadores ${ }^{17}$.

No se han utilizado el resto de preguntas del cuestionario de Kourinka referidas a las características del dolor, su duración, su intensidad, la necesidad de reubicación, el tratamiento recibido a consecuencia del mismo etc. dado que eran ajenas a los objetivos de nuestro estudio.

Entre los riesgos psicosociales identificados destaca la exigencia de nivel de atención elevado en población laboral general. En la población navarra según la II Encuesta Navarra de Salud y Condiciones de Trabajo (II ENSyCT) esta atención elevada afectaba al $62,7 \%$ mientras alcanza al $80,9 \%$ de la población de nuestro estudio. También son mayores las frecuencias de trabajadoras expuestas a ritmos elevados de trabajo y realización de tareas muy repetitivas de corta duración en nuestro estudio. En esta misma publicación se encuentra que en España tanto la frecuencia de trabajadores que requerían mantener el nivel de atención elevado, un alto ritmo de trabajo y una elevada cantidad de trabajo eran significativamente mayores que en Navarra. Estos resultados que asocian el nivel de riesgo a la percepción de realización de elevada actividad física coinciden con otros estudios ${ }^{18}$.

La segmentación corporal donde se ubican los daños percibidos establecida en el Cuestionario de Kourinka es distinta a la de otros estudios revisados. En nuestro estudio la espalda constituye un segmento mientras en otros estudios se distingue la parte alta de la misma de la zona lumbar. Esto dificulta las comparaciones. En la II ENSyCT la mayor prevalencia de dolor se presenta en la zona lumbar mientras en nuestro estudio lo hace en la zona del cuello. Si sumamos las frecuencias de cuello y parte alta de la espalda de la II Encuesta Navarra encontramos que sería la zona leader en cuanto a frecuencia de molestias con un 56,3 seguida de la zona lumbar con el 39,1\%. En nuestro estudio las molestias en la zona del cuello alcanzan casi a 2/3 de la población. Estos resultados son coherentes con el tipo de exposición ergonómica a movimientos repetidos de extremidad superior y manipulación manual de cargas no muy elevadas de manera frecuente que se dan en el trabajo de esterilización hospitalaria.

Tras descartar por observación directa diferencias de diseño de puesto, la distinta percepción de exposición a diversas variables de riesgo entre el Hospital San Pedro y el Complejo Hospitalario de Navarra (tabla IV), puede asociarse a las tareas de lavado que se realizan en el primero de los hospitales y no se llevan a efecto en el Hospital B del Complejo cuya plantilla representa el 64,29 \% de la muestras navarra. La prevalencia de daños sin embargo no es diferente según hospitales excepto en la parte izquierda del segmento hombro-brazo izquierdo siendo mayor en el Complejo navarro. Esta diferencia no parece ser explicada por la mayor edad media de la plantilla de navarra ya que dichos daños no se asocian a la edad en nuestro estudio.

También las trabajadoras con contrato temporal perciben tener unas peores condiciones de trabajo en cuanto a las variables ergonómicas y psicosociales aunque esta percepción no se refleja en una diferente prevalencia de daños. Otros estudios muestran un menor nivel de estrés en trabajadores temporales ${ }^{19}$. La inestabilidad en el empleo y la turnicidad en la jornada formarían parte de la falta de compensaciones del modelo de riesgo psicosocial de Siegrist.

Las molestias de cuello se van a asociar con la edad menor de 45 años, el trabajo a turnos y la percepción de trabajar con rapidez. También el dolor de espalda se asocia al ritmo de trabajo y la menor categoría de edad. La plantilla más joven percibe también una mayor cantidad de trabajo a realizar y una menor disponibilidad de espacio para realizar movimientos necesarios. La velocidad de trabajo, la presión de tiempos son variables componentes de los riesgos psicosociales tanto en el modelo de Karasek et $\mathrm{Al}^{20}$ cuando se refiere a exigencias ${ }^{21}$. Como en el de Siegrist et $\mathrm{Al}^{22}$ cuando hablamos de demanda. La constatación de su papel en la explicación de la probabilidad de daño ha conducido a la inclusión de este tipo de factores entre los que se consideran en alguno de los métodos 
más actuales de evaluación del riesgo como el cálculo del Índice OCRA para evaluar el riesgo de movimientos repetidos en términos de frecuencia de movimientos y ritmo impuesto por la tarea o máquina ${ }^{23}$. En nuestro estudio se refleja la importancia de algunas de las variables psicosociales a la hora de explicar la aparición de los daños ergonómicos. En algún estudio se observa incluso asociación entre exposición psicosocial y dolor de cuello-nuca y no asociación con la actividad física ${ }^{24}$. No obstante otros estudios asocian la mayor edad con el aumento del riesgo de lesión osteo muscular ${ }^{15}$.

La mayor prevalencia de MME se presenta en cuello y espalda, lo cual coincide con los resultados del estudio realizado por M. $^{a}$ S. Gallón ${ }^{25}$ y además se sabe que este padecimiento también se puede presentar en diversos colectivos de trabajadores que realizan actividades de posturas forzadas, movimientos repetidos y en profesionales como odontólogos debido a su actividad clínica como lo señala Chávez López $\mathrm{R}^{26}$.

En otro estudio realizado en profesionales sanitarios en las islas Baleares en España revela que el 59,3\% de los trabajadores presentaban dolor de espalda y el 26,7\% dolor cervical $^{12}$, resultados que no coinciden con nuestro estudio en el que en primer lugar lo ocupa las molestias en el cuello. Además las prevalencias encontradas en nuestro estudio son mayores que en aquel.

La turnicidad se asocia a mayores índices de molestias en cuello y hombro derecho. La turnicidad también aparece asociada a mayores requerimientos de forzar la vista por motivos de iluminación y a una menor autonomía para establecer el orden de las tareas.

Aunque el mayor nivel de estudios aparece ligado a una mayor necesidad de atención a la hora de realizar la tarea y una menor posibilidad de realización de los movimientos necesarios, la no asociación de esta variable a los índices de daños puede indicarnos que estamos ante el fruto de las múltiples comparaciones realizadas, ante un artefacto estadístico.

Recientes estudios muestran que puede haber incluso diferencias en la coordinación y estrategias motoras durante trabajos que implican tareas de miembro superior. También parece haber diferencias de percepción de las exposiciones psicosociales $^{18}$.

Las trabajadoras perciben estar expuestas particularmente a un exceso de carga de trabajo asociada a la atención requerida por la tarea, las presiones de tiempo, identificadas por el trabajo muy rápido y el tiempo insuficiente, y a la ausencia de autonomía a la hora de organizar su propio trabajo o al no poder poner en práctica sus propias ideas. Sienten también contar con escaso apoyo social, tanto de compañeras como de supervisoras, e identifican estar expuestas a riesgos ergonómicos asociado al diseño del puesto de trabajo que les limitan el trabajar con comodidad, la libertad de movimientos, el no tener que forzar la vista y el adoptar posturas adecuadas.

\section{CONCLUSIONES}

1. El colectivo de trabajadoras de las unidades de esterilización hospitalaria está especialmente expuesto a factores de riesgo que aumentan la probabilidad de padecer molestias y daños músculo-esqueléticos.

2. Los movimientos repetidos de extremidades superiores y la manipulación manual de cargas destacan entre los riesgos ergonómicos y las exigencias de atención, los altos ritmos de trabajo, los plazos cortos y la falta de apoyo social entre los riesgos psicosociales a los que está expuesto este colectivo.

3. Las trabajadoras de esterilización de hospitales presenta altas tasas de prevalencia de molestias.

4. Entre las regiones anatómicas afectadas por las molestias destaca el cinturón formado por el cuello-hombros y la espalda. 
5. Sería oportuna la realización en este colectivo de la evaluación de riesgos de movimientos repetidos en extremidad superior con métodos validados como el OCRA.

6. La elevada prevalencia a temporalidad en el empleo requeriría intervenciones correctoras al respecto.

7. Así mismo las exposiciones ergonómicas y psicosociales identificadas aconsejan actuaciones preventivas sobre todo en los colectivos en los que su frecuencia es mayor como el Hospital San Pedro, el personal eventual, las trabajadoras menores de 45 años y la plantilla que trabaja a turnos.

8. Los estudios sobre daño ergonómico, sean descriptivos o causales, deben integrar los riesgos psicosociales, tanto organizativos como relacionales, entre las variables a investigar dado que aparecen sólidamente asociados a los daños músculoesqueléticos.

9. Sería de interés incluir variables de compensación del modelo de Siegrist ente los factores de riesgo en futuros estudios de asociación entre exposición psicosocial y efectos ergonómicos.

\section{AGRADECIMIENTO}

Nuestro primer agradecimiento y reconocimiento es para el Dr. Iñaki Moreno Sueskun, Médico especialista en Medicina de Trabajo de la Sección de Epidemiología, Investigación y Evaluación Sanitaria en el Instituto de Salud Pública y Laboral de Navarra y tutor del proyecto. Por su enorme implicación en el estudio y su permanente disposición para poder llegar hasta el final de nuestro proyecto.

Agradecemos la valiosa colaboración de las trabajadoras del servicio de esterilización que han participado desinteresadamente en las encuestas.

A la Dra. Silvia Santo Domingo por su colaboración desinteresada.

A nuestras coordinadoras de residencia Dra. Carmen Arceiz y Dra. Vega García.

\section{REFERENCIAS BIBLIOGRÁFICAS}

1. Las enfermedades músculo esqueléticas. TME de origen laboral. [acceso 13/08/2010]. Disponible en: http://salud.discapnet.es/castellano/Salud/Prevencion/Riesgos/Enfermedades/Paginas/E_L_ enfermedades_2.

2. Riso S. El efecto de los cambios en el trabajo en el resurgimiento de los problemas musculoesqueléticos. Magazine [revista en internet] 2007 [acceso 23 de Agosto de 2010]; [3-7]. Disponible en: http://osha. europa.eu/publications/megazine/10.

3. Ministerio de Sanidad y Consumo. Protocolo de Vigilancia Sanitaria específica sobre movimientos repetidos. Madrid: Centro de publicaciones del ministerio de sanidad y consumo. 2000.

4. National Institute for Occupational Safety and Health Centers for Disease control and Prevention. Linda Rosenstock, M.D;M.P.H. Musculoskeletal Disorders and workplace factors.

5. Eransus J Díez de Ulzurrun M. et al. Los trastornos músculo-esqueléticos. Una prioridad en la atención preventiva en la C.A. de Navarra. La mutua [revista en internet] 2010 [acceso 03 Agosto 2010]; 17(7). Disponible en: http://www.fraternidad.com/es-ES/revista/REVISTA-LA- MUTUA-NUMERO-17_17.html.

6. VI Encuesta Nacional de Condiciones de Trabajo. [acceso 05/08/2010]. Disponible en: http://www.oect. es/Observatorio/Contenidos/InformesPropios/Desarrollados/Ficheros.

7. Duque I, Zuluaga D, Pinilla A. Prevalencia de Lumbalgia y factores de riesgo en enfermeros y auxiliares de la ciudad de Manizales, Colombia. Hacia la promoción de la salud [Revista en internet] 2011 [Acceso 30 de Enero 2014; [33]. Disponible en: http://promocionsalud.ucaldas.edu.co/downloads/Revista16(1)3.

8. Agencia Europea para la Seguridad y Salud en el Trabajo. Los TME de origen laboral en los estados miembros de la Unión Europea: inventario de factores socioeconómicos 2000. Disponible en: http://osha. europea.eu/es/publications/factsheets/9. 
9. Morata M, Ferrer V. Interacción entre estrés ocupacional, estrés psicológico y dolor lumbar: un estudio en profesionales sanitarios de Traumatología y cuidados intensivos. [revista en internet]* 2004 [acceso 29 de Agosto 2010]; 15 (3):[199-211]. Disponible en: http://fundacionmapfre.com/ccm/content/documentos/ fundacion/salud/revista-medicina/vol15-n3-art5.

10. Peinador R. Manipulación manual de cargas y trabajo de enfermería. Revista científica y de divulgación [revista en internet] 2005 [acceso 20 de Agosto 2010]. (3). Disponible en: http://www.ee.isics.es/servlet?P agename $=$ ExcelenciaEnfermera/Articulo.EE/plantilla_articulo_EE.

11. Valecillo M, Quevedo A, Lubo A, Dos Santos A, Montiel M, Carmejo M. Síntomas músculo-esqueléticos y estrés laboral en el personal de enfermería de un hospital militar. [revista en Internet]* 2009 juliodiciembre. [acceso 3 de octubre 2010]; 17 (2): 85-95. Disponible en: www.scielo.org.ve/scielo. php?script=sci_arttext $\&$ pid=S1315-0138.

12. Siniestralidad Laboral en Navarra año 2010 [sede web]. Navarra: Instituto Navarro de Salud Laboral,; 2011 [acceso 30 de Julio 2012]. Enfermedades profesionales en Navarra 2010 [1 página]. Disponible en: http:// www.navarra.es/NR/rdonlyres/DB78932D.

13. Observatorio de enfermedades profesionales (CEPROSS) y de enfermedades causadas o agravadas por el trabajo (PANOTRATSS). Informe anual 2011. [acceso 15 de Agosto 2012]. Disponible en: www.segsociales.es/prdi00/groups.

14. Troconi F, Lubo A, Montiel M, Quevedo A, Rojas L, Chacin B. Valoración postural y riesgo de lesión músculo esquelética en trabajadores de una plataforma perforación petrolera. [revista en Internet]* 2008 enero-jun. [acceso 19 de noviembre 2011; 16(1), 29-38. Disponible en: www.scielo.org.ve/scielo. php?pid=s1315-0138.

15. Kuorinka I, Jonsson B, Kilbom A, Vinterberg H, Biering F, et al. Standardised Nordic questionnaires for the analysis of musculoskeletal symptoms. Applied ergonomics 1987, 18.3, 233-237.

16. Côté JN. A critical review on physical factors and functional characteristics that may explain a sex/gender difference in work-related neck/shoulder disorders. Ergonomics. 2012;55(2):173-82. doi: 10.1080/00140139.2011.586061. Epub 2011 Aug 17.

17. Palmer KT, Syddall H, Cooper C, Coggon D. Smoking and musculoskeletal disorders: findings from a British national survey Ann Rheum Dis.2003 Jan;62(1):33-6.

18. Bodin J., Ha C., Sérazin C., Descatha A.,Leclerc A., Goldberg M. Roquelaure Y. Effects of individual and work-related factors on incidence of shoulder pain in a large working population.[Internet: 2012 mayo: https://www.jstage.jst.go.jp/article/joh/advpub/0/advpub_11-0262-OA/_pdf]

19. Benach J. Gimeno D. Benavides FG. Types of employement and health in the European Union. European Foundation for the Improvement of Living and Work Conditions. 2002. www.eurofound.eu.int.

20. Karasek R. Theorell T. Healthy Work, stress, productivity and the reconstruction of working life. New York NY Basic Books 1990

21. Johnson JV, Hall EM. Job strain, workplace, social support and cardiovascular disease: A cross sectiona study of a random sample of the swedish working population. Am J Public health 1988; 78: 1336-1342.

22. Siegrist J, Peter R, Cremer P, Siedel D. Chronic work stress is associated with atheroetic lipids and elevated fibrinogen in middle-aged men. J Internal Medicine 1997: 242: 149-56.

23. Colombini D. Occipenti E, Grecco A. Risk asessment and management of repetitive movements and exertions of upper limbs. 2002 Elsevier pp 111-117.

24. Palmer KT,Walker-Bone K, Griffin MJ, Syddall H, Pannett B, Coggon D, Cooper C Prevalence and occupational associations of neck pain in the British population. Scand J Work Environ Health. 2001 Feb;27(1):49-56.

25. Gallón M, Estrada Jinna, Quintero Marieth, Carvajal Reynaldo, Velazquez Juan. Prevalencia de Síntomas Osteomusculares en trabajadores de Oficina de una Empresa de Consultoría en Ingeniería Eléctrica de Cali Colombia. [revista en internet]* 201 Enero. [acceso 19 de Noviembre de 2011]; 1 (1). Disponible en: http://www.unilibrecali.edu.co/Revista_Colombiana_Salud_Ocupacional/pdf.

26. Chávez R, Preciado M, Colunga C, Mendoza P, Aranda C. Trastornos musculoesqueléticos en Odontólogos de una institución pública de Guadalajara, México. [revista en internet]* 2009 Julio-Septiembre. [acceso 19 de Noviembre de 2011]; (33). Disponible en: http://www.cienciaytrabajo.cl/pdf/33/paginas152.pdf.

27. Llopis Pérez J. La estadística: una orquesta hecha instrumento [Sede web]. Barcelona: 2013; acceso 20 Enero 2014. Disponible en: http://estadisticaorquestainstrumento.wordpress.com/2012/12/16. 


\section{ANEXO}

\section{Cuestionario}

Encuesta $\mathbf{n}^{\circ}$

1.- Edad (años)

2.- Sexo 1- mujer

2- Hombre

3.- Nivel escolar/Formación: 1- ESO

2- Bachiller o FP

3- Diplomatura/Superior

4.- Centro de Trabajo: 1- Complejo hospitalario de Navarra:

A $\square \quad B$

\section{2- Hospital San Pedro}

5.- Contrato 1- fijo

2- Temporal

6.- Antigüedad en el puesto (años)

7.- Otros puestos de trabajo en el último año

8.- Cuánto tiempo lleva trabajando en la empresa actual? (años)

(meses)

\section{A continuación rodee con un círculo el número correspondiente a la respuesta} seleccionada

9.- ¿Qué días de la semana trabaja habitualmente?

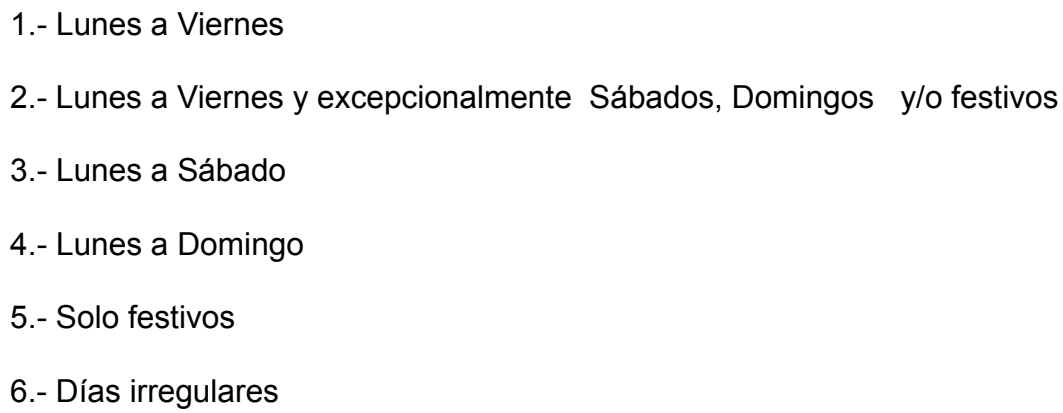

10.- ¿Qué tipo de jornada u horario de trabajo tiene habitualmente?

1.- Jornada partida (mañana y tarde)

2.- Turno fijo/jornada continuada de mañana

3.- Turno fijo/jornada continuada de tarde 


\section{4.- Turno fijo/jornada continuada de noche \\ 5.- Turnos rotatorios excepto el de noche \\ 6.- Turnos rotatorios con el de noche \\ 7.- Horarios irregulares}

11.- En los últimos tres meses, ¿Ha tenido molestias en.....?

\begin{tabular}{|l|l|l|}
\hline & NO & SI \\
\hline 1- Cuello & & \\
\hline 2- Hombro derecho & & \\
\hline 3- Hombro izquierdo & & \\
\hline 4- Espalda & & \\
\hline 5- Codo-Antebrazo derecho & & \\
\hline 6- Codo-Antebrazo Izquierdo & & \\
\hline 7- Mano-Muñeca derecha & & \\
\hline 8- Mano-Muñeca Izquierdo & & \\
\hline
\end{tabular}

Si todas sus respuestas a la pregunta anterior han sido "NO", no continue cumplimentando el cuestionario y entréguelo.

12.- ¿Desde hace cuánto tiempo ha presentado molestias? Señale desde hace cuanto que presenta molestias, para cada segmento corporal afectado.

\begin{tabular}{|l|c|c|c|c|c|}
\hline & 1 & 2 & 3 & 4 & 5 \\
\cline { 2 - 6 } & 1 mes o menos & $\begin{array}{c}\text { De 2 a 3 } \\
\text { meses }\end{array}$ & $\begin{array}{c}\text { De 4 a 6 } \\
\text { meses }\end{array}$ & $\begin{array}{c}\text { De 7 a 9 } \\
\text { meses }\end{array}$ & $\begin{array}{c}10 \text { a 12 } \\
\text { meses }\end{array}$ \\
\hline 1- Cuello & & & & & \\
\hline 2- Hombro derecho & & & & & \\
\hline 3- Hombro izquierdo & & & & & \\
\hline 4- Espalda & & & & & \\
\hline 5- Codo-Antebrazo derecho & & & & & \\
\hline 6- Codo-Antebrazo izquierdo & & & & \\
\hline 7- Mano-Muñeca derecha & & & & & \\
\hline 8- Mano-Muñeca izquierda & & & & & \\
\hline
\end{tabular}


13.- ¿Ha tenido que cambiar de puesto de trabajo a causa de molestias en...?

\begin{tabular}{|l|l|l|}
\hline & NO & SI \\
\hline 1- Cuello & & \\
\hline 2- Hombro derecho & & \\
\hline 3- Hombro izquierdo & & \\
\hline 4- Espalda & & \\
\hline 5- Codo-Antebrazo derecho & & \\
\hline 6- Codo-Antebrazo izquierdo & & \\
\hline 7- Mano-Muñeca derecha & & \\
\hline 8- Mano-Muñeca izquierda & & \\
\hline
\end{tabular}

14.- ¿Cuánto tiempo han durado sus molestias en los últimos 3 meses? Señale la duración de sus molestias para cada segmento corporal afectado.

\begin{tabular}{|l|c|c|c|c|}
\hline & 1 & 2 & 3 & 4 \\
\cline { 2 - 5 } & 1 a 3 días & $\begin{array}{c}\text { 8 a 30 } \\
\text { días }\end{array}$ & $\begin{array}{c}\text { > 30 días } \\
\text { continuo }\end{array}$ & Permanente \\
\hline 1- Cuello & & & & \\
\hline 2- Hombro derecho & & & & \\
\hline 3- Hombro izquierdo & & & & \\
\hline 4- Espalda & & & & \\
\hline 5- Codo-Antebrazo derecho & & & & \\
\hline 6- Codo-Antebrazo izquierdo & & & & \\
\hline 7- Mano-Muñeca derecha & & & & \\
\hline 8- Mano-Muñeca izquierda & & & & \\
\hline
\end{tabular}

15.- ¿Cuánto dura cada episodio de dolor? Señale la duración de sus crisis de dolor.

\begin{tabular}{|l|c|c|c|c|c|}
\hline & 1 & 2 & 3 & 4 & 5 \\
\cline { 2 - 6 } & $\begin{array}{c}<1 \\
\text { hora }\end{array}$ & $\begin{array}{c}\text { 1 a 24 } \\
\text { horas }\end{array}$ & $\begin{array}{c}\text { 1 a 7 } \\
\text { días }\end{array}$ & $\begin{array}{c}\text { 1 a 4 } \\
\text { semanas }\end{array}$ & $\begin{array}{c}>1 \\
\text { mes }\end{array}$ \\
\hline 1- Cuello & & & & & \\
\hline 2- Hombro derecho & & & & & \\
\hline 3- Hombro izquierdo & & & & & \\
\hline 4- Espalda & & & & & \\
\hline 5- Codo-Antebrazo derecho & & & & & \\
\hline 6- Codo-Antebrazo izquierdo & & & & & \\
\hline 7- Mano-Muñeca derecha & & & & & \\
\hline 8- Mano-Muñeca izquierda & & & & & \\
\hline
\end{tabular}


16.- ¿Cuánto tiempo estas molestias le han impedido hacer su trabajo en los últimos 3 meses? Señale la duración de su incapacidad temporal.

\begin{tabular}{|l|c|c|c|c|}
\hline & 1 & 2 & 3 & 4 \\
\cline { 2 - 5 } & 0 día & $\begin{array}{c}\text { 1 a 7 } \\
\text { días }\end{array}$ & $\begin{array}{c}\text { 1 a 4 } \\
\text { semanas }\end{array}$ & $>$ 1 mes \\
\hline 1- Cuello & & & & \\
\hline 2- Hombro derecho & & & & \\
\hline 3- Hombro izquierdo & & & & \\
\hline 4- Espalda & & & & \\
\hline 5- Codo-Antebrazo derecho & & & & \\
\hline 6- Codo-Antebrazo izquierdo & & & & \\
\hline 7- Mano-Muñeca derecha & & & & \\
\hline 8- Mano-Muñeca izquierda & & & & \\
\hline
\end{tabular}

17.- ¿Ha recibido tratamiento por estas molestias en los últimos 3 meses?

\begin{tabular}{|l|l|l|}
\hline & NO & SI \\
\hline 1- Cuello & & \\
\hline 2- Hombro derecho & & \\
\hline 3- Hombro izquierdo & & \\
\hline 4- Espalda & & \\
\hline 5- Codo-Antebrazo derecho & & \\
\hline 6- Codo-Antebrazo izquierda & & \\
\hline 7- Mano-Muñeca derecha & & \\
\hline 8- Mano-Muñeca izquierda & & \\
\hline
\end{tabular}

18.- ¿Ha tenido molestias los últimos 7 días?

\begin{tabular}{|l|l|l|}
\hline & \multicolumn{1}{|c|}{ NO } & SI \\
\hline 1- Cuello & & \\
\hline 2- Hombro derecho & & \\
\hline 3- Hombro izquierdo & & \\
\hline 4- Espalda & & \\
\hline 5- Codo-Antebrazo derecho & & \\
\hline 6- Codo-Antebrazo izquierdo & & \\
\hline 7- Mano-Muñeca derecha & & \\
\hline 8- Maño-Muñeca izquierda & & \\
\hline
\end{tabular}


19.- Califique la intensidad de sus molestias en los últimos 7 días

\begin{tabular}{|l|l|l|l|l|}
\hline & $\begin{array}{c}1=\text { eve } \\
\text { molestias }\end{array}$ & $\begin{array}{c}\text { 2=moderada } \\
\text { molestias }\end{array}$ & $\begin{array}{c}3=\text { fuertes } \\
\text { molestias }\end{array}$ & $\begin{array}{c}\text { 4= molestias } \\
\text { muy fuertes }\end{array}$ \\
\hline 1- Cuello & & & & \\
\hline 2- Hombro derecho & & & & \\
\hline 3- Hombro izquierdo & & & & \\
\hline 4- Espalda & & & & \\
\hline 5- Codo-Antebrazo derecho & & & & \\
\hline 6- Codo-Antebrazo izquierdo & & & & \\
\hline 7- Mano-Muñeca derecha & & & & \\
\hline 8- Mano-Muñeca izquierda & & & & \\
\hline
\end{tabular}

20.- Señale a qué atribuye estas molestias

21.- En su puesto de trabajo, ¿Con qué frecuencia su tarea requiere realizar alguna de las siguientes actividades?

\begin{tabular}{|l|c|c|c|c|c|}
\hline & 1 & 2 & 3 & 4 & 5 \\
\cline { 2 - 5 } & Siempre & $\begin{array}{c}\text { Muchas } \\
\text { veces }\end{array}$ & $\begin{array}{c}\text { Algunas } \\
\text { veces }\end{array}$ & $\begin{array}{c}\text { Solo alguna } \\
\text { vez }\end{array}$ & Nunca \\
\hline 1- Manipular cargas & & & & & \\
\hline $\begin{array}{l}\text { 2- Realizar movimientos } \\
\text { repetidos }\end{array}$ & & & & & \\
\hline $\begin{array}{l}\text { 3- Realizar posturas } \\
\text { forzadas }\end{array}$ & & & & & \\
\hline 4- Aplicar fuerzas & & & & & \\
\hline
\end{tabular}

22.- En su puesto de trabajo, con qué frecuencia el espacio del que dispone le permite....

\begin{tabular}{|l|c|c|c|c|c|}
\hline & 1 & 2 & 3 & 4 & 5 \\
\cline { 2 - 6 } & Siempre & $\begin{array}{c}\text { Muchas } \\
\text { veces }\end{array}$ & $\begin{array}{c}\text { Algunas } \\
\text { veces }\end{array}$ & $\begin{array}{c}\text { Solo alguna } \\
\text { vez }\end{array}$ & Nunca \\
\hline 1- Trabajar con comodidad & & & & & \\
\hline $\begin{array}{l}\text { 2- Poder realizar los } \\
\text { movimientos necesarios }\end{array}$ & & & & & \\
\hline 3- Cambiar de posturas & & & & & \\
\hline
\end{tabular}


23.- En su puesto de trabajo, con qué frecuencia la iluminación le permite....

\begin{tabular}{|l|c|c|c|c|c|}
\hline & 1 & 2 & 3 & 4 & 5 \\
\cline { 2 - 6 } & Siempre & $\begin{array}{c}\text { Muchas } \\
\text { veces }\end{array}$ & $\begin{array}{c}\text { Algunas } \\
\text { veces }\end{array}$ & $\begin{array}{c}\text { Solo alguna } \\
\text { vez }\end{array}$ & Nunca \\
\hline $\begin{array}{l}\text { 1-Trabajar en una } \\
\text { postura adecuada }\end{array}$ & & & & \\
\hline 2- No forzar la vista & & & & & \\
\hline
\end{tabular}

24.- En su puesto de trabajo, con qué frecuencia debe....

\begin{tabular}{|l|c|c|c|c|c|}
\hline & 1 & 2 & 3 & 4 & 5 \\
\cline { 2 - 5 } & Siempre & $\begin{array}{l}\text { Muchas } \\
\text { veces }\end{array}$ & $\begin{array}{c}\text { Algunas } \\
\text { veces }\end{array}$ & $\begin{array}{c}\text { Solo alguna } \\
\text { vez }\end{array}$ & Nunca \\
\hline $\begin{array}{l}\text { 1- Mantener un nivel de } \\
\text { atención alto o muy alto }\end{array}$ & & & & & \\
\hline $\begin{array}{l}\text { 2- Atender a varias } \\
\text { tareas al mismo tiempo }\end{array}$ & & & & & \\
\hline $\begin{array}{l}\text { 3- Realizar tareas } \\
\text { complejas, complicadas } \\
\text { o difíciles }\end{array}$ & & & & & \\
\hline $\begin{array}{l}\text { 4- Esconder sus propias } \\
\text { emociones en el puesto } \\
\text { de trabajo }\end{array}$ & & & & & \\
\hline $\begin{array}{l}\text { 5- Considera su trabajo } \\
\text { excesivo }\end{array}$ & & & & & \\
\hline
\end{tabular}

25.- En su puesto de trabajo, con qué frecuencia es necesario....

\begin{tabular}{|l|c|c|c|c|c|}
\hline & 1 & 2 & 3 & 4 & 5 \\
\cline { 2 - 6 } & Siempre & $\begin{array}{c}\text { Muchas } \\
\text { veces }\end{array}$ & $\begin{array}{c}\text { Algunas } \\
\text { veces }\end{array}$ & $\begin{array}{c}\text { Solo alguna } \\
\text { vez }\end{array}$ & Nunca \\
\hline 1- Trabajar muy rápido & & & & & \\
\hline estrictos o muy cortos & & & & & \\
\hline $\begin{array}{l}\text { 3- Tener tiempo suficiente } \\
\text { para realizar su trabajo }\end{array}$ & & & & & \\
\hline
\end{tabular}


26.- En su puesto de trabajo, con qué frecuencia.....

\begin{tabular}{|l|c|c|c|c|c|}
\hline & 1 & 2 & 3 & 4 & 5 \\
\cline { 2 - 5 } & Siempre & $\begin{array}{c}\text { Muchas } \\
\text { veces }\end{array}$ & $\begin{array}{c}\text { Algunas } \\
\text { veces }\end{array}$ & $\begin{array}{c}\text { Solo alguna } \\
\text { vez }\end{array}$ & Nunca \\
\hline $\begin{array}{l}\text { 1- Dispone de } \\
\text { informaciones claras y } \\
\text { suficientes para realizar } \\
\text { correctamente su trabajo }\end{array}$ & & & & & \\
\hline $\begin{array}{l}\text { 2- Recibe información y } \\
\text { adiestramiento por parte } \\
\text { de la empresa }\end{array}$ & & & & & \\
\hline $\begin{array}{l}\text { 3- Los horarios los fija la } \\
\text { empresa sin posibilidad de } \\
\text { cambio }\end{array}$ & & & & & \\
\hline $\begin{array}{l}\text { 4- Tiene libertad para } \\
\text { decidir vacaciones/días } \\
\text { libres }\end{array}$ & & & & & \\
\hline $\begin{array}{l}\text { 5- Tiene oportunidad de } \\
\text { hacer aquello que sabe } \\
\text { hacer mejor }\end{array}$ & & & & & \\
\hline $\begin{array}{l}\text { 6- Puede poner en práctica } \\
\text { sus propias ideas en su } \\
\text { trabajo }\end{array}$ & & & & & \\
\hline
\end{tabular}

27.- En su puesto de trabajo, con qué frecuencia tiene autonomía en.....

\begin{tabular}{|l|c|c|c|c|c|}
\hline & 1 & 2 & 3 & 4 & 5 \\
\cline { 2 - 6 } & Siempre & $\begin{array}{c}\text { Muchas } \\
\text { veces }\end{array}$ & $\begin{array}{c}\text { Algunas } \\
\text { veces }\end{array}$ & $\begin{array}{c}\text { Solo alguna } \\
\text { vez }\end{array}$ & Nunca \\
\hline 1- El orden de las tareas & & & & & \\
\hline 2- El método de trabajo & & & & & \\
\hline 3- El ritmo de trabajo & & & & & \\
\hline $\begin{array}{l}\text { 4- La distribución y/o } \\
\text { duración de las pausas } \\
\text { en el trabajo }\end{array}$ & & & & & \\
\hline
\end{tabular}

28.- En su puesto de trabajo, con qué frecuencia puede.....

\begin{tabular}{|l|c|c|c|c|c|}
\hline & 1 & 2 & 3 & 4 & 5 \\
\cline { 2 - 6 } & Siempre & $\begin{array}{l}\text { Muchas } \\
\text { veces }\end{array}$ & $\begin{array}{c}\text { Algunas } \\
\text { veces }\end{array}$ & $\begin{array}{c}\text { Solo alguna } \\
\text { vez }\end{array}$ & Nunca \\
\hline $\begin{array}{l}\text { 1- Obtener ayuda de } \\
\text { compañeros si la pide }\end{array}$ & & & & & \\
\hline $\begin{array}{l}\text { 2- Obtener ayuda de } \\
\text { supervisores/jefes si la } \\
\text { pide }\end{array}$ & & & & & \\
\hline $\begin{array}{l}\text { 3- Las relaciones } \\
\text { personales son positivas }\end{array}$ & & & & & \\
\hline
\end{tabular}

